\title{
Lenalidomide Plus Dexamethasone is an Active Rescue Treatment for Myeloma with Severe Renal Impairment Failing to Bortezomib- Based Induction Therapy
}

\author{
Giuseppe Mele* and Domenico Pastore \\ Haematology and BMT Unit, A. Perrino Hospital, Brindisi, Italy
}

*Corresponding author: Giuseppe Mele, Haematology and BMT Unit, Antonio Perrino Hospital, SS 7 per Mesagne, 72100 Brindisi, Italy, Tel: +39-831-537506, Fax: +39-831-537513, E-mail: giuseppemele2007@gmail.com

\begin{abstract}
Patients with relapsed/refractory Multiple Myeloma (rrMM) and End Stage Renal Disease (ESRD) requiring hemodialysis must receive lenalidomide at a dosage of $5 \mathrm{mg}$ daily on days 1-21 of repeated 28-day cycles, due to substantial elimination via the kidneys. Unfortunately, despite dose reduction, severe adverse events occur with a high frequency. Therefore, lenalidomide is recommended mainly in patients with mild-to-moderate renal impairment. In our experience, different lenalidomide dose adjustment (5 mg every other day on days 1-21 of a 28-day cycle after dialysis) from the recommendations of the manufacturer was chosen due to additional fragilities (serious comorbidities, poor performance status or/and advanced age). We observed a lower-than-expected incidence of haematological toxicities without an excess of prolonged cytopenia despite long-term exposure to lenalidomide.
\end{abstract}

\section{Keywords}

Myeloma, End stage renal disease, Hemodialysis, Lenalidomide

\section{Introduction}

The combination of "lenalidomide plus dexamethasone" is an effective option for the treatment of patients with relapsed/refractory Multiple Myeloma ( $r \mathrm{rMM}$ ). Since lenalidomide is primarily excreted unchanged by the kidney, adjustments to the starting dose are recommended to provide appropriate drug exposure in patients with moderate or severe renal impairment [1]. Particularly, patients on hemodialysis (Creatinine Clearance $[\mathrm{CLCr}]<30 \mathrm{~mL} / \mathrm{min}$ requiring dialysis) must receive lenalidomide at a dosage of $5 \mathrm{mg}$ daily on days 1-21 of a 28-day cycle, after dialysis [2]. There are limited experiences in patients with renal impairment who received lenalidomide because of severe adverse events that occur with a high frequency in patients with $\mathrm{MM}$ requiring hemodialysis treated with this drug $[3,4]$. In addition, the interpretation of clinical data is limited by the heterogeneity of the dosages chosen by the treating physician with regard to individual clinical circumstances. De la Rubia, et al., in a multicenter retrospective study, evaluated 15 patients with relapsed MM and severe renal impairment requiring hemodialysis; grade $3 / 4$ neutropenia was the most common side effect $(53 \%$ of the patients) and severe infections were a major cause of morbidity and mortality requiring additional dose reduction of lenalidomide and G-CSF prophylaxis [3]. Oehrlein, et al. conducted a retrospective study of 26 patients of whom 6 were on dialysis; grade $3 / 4$ thrombocytopenia was the most common side effect (66.7\%) reported in patients who required dialysis [4].

Here we evaluate a divergent lenalidomide dose adjustment from the recommendations as a treatment for patients with light chain-induced renal failure. Our data suggest that "lenalidomide plus dexamethasone" is an active rescue treatment also for patients with relapsed MM and End Stage Renal Disease (ESRD) requiring dialysis after the failure of bortezomib-based induction therapy.

\section{Patients and Methods}

We reported our retrospective experience of 4 pa- 
Table 1: Characteristic Patients.

\begin{tabular}{|c|c|}
\hline Median (range) Age, y & $73.5(62-77)$ \\
\hline ANC $\times 10^{3} /$ uL (range) & $2.9(2.8-3.3)$ \\
\hline Hgb g/dL (range) & $12(9.3-12.6)$ \\
\hline Platelet $\times 10^{3} /$ uL (range) & $124(59-225)$ \\
\hline CLCr ml/min (range) & Not required \\
\hline 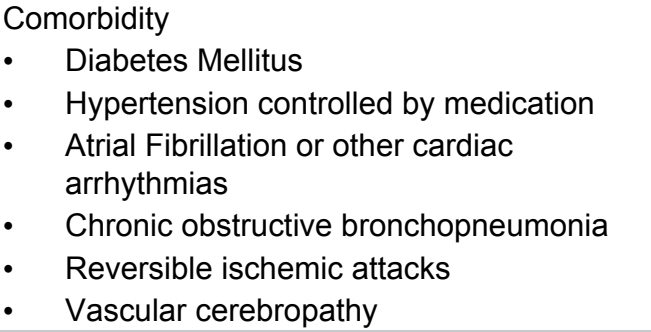 & $\begin{array}{l}\text { yes } \\
\text { yes } \\
\text { yes } \\
\text { yes } \\
\text { yes } \\
\text { yes }\end{array}$ \\
\hline Median number of prior therapies & $1(1-2)$ \\
\hline Median number of lenalidomide cycles & $\begin{array}{l}12(6-19 \\
\text { cycles) }\end{array}$ \\
\hline $\begin{array}{l}\text { ORR with previous treatments (n. patients) } \\
\text { - } \quad \text { PR } \\
\text { - } \quad \text { VGPR } \\
\text { - } \quad \text { CR }\end{array}$ & $\begin{array}{l}4 \\
3 \\
1 \\
0\end{array}$ \\
\hline $\begin{array}{l}\text { ORR with "lenalidomide + dexamethasone" (n. } \\
\text { patients) } \\
\text { - PR } \\
\text { - } \quad \text { VGPR } \\
\text { - } \quad \text { CR }\end{array}$ & $\begin{array}{l}4 \\
2 \\
1 \\
1\end{array}$ \\
\hline Median duration of response, months (range) & $13.5(6-21)$ \\
\hline $\begin{array}{l}\text { Non-Hematological Toxicities (grade } \geq 3 \text { ) } \\
\text { - Diarrhea } \\
\text { - Constipation } \\
\text { - Thromboembolic events } \\
\text { - Infections }\end{array}$ & $\begin{array}{l}0 \\
0 \\
0 \\
0 \\
0\end{array}$ \\
\hline $\begin{array}{l}\text { Hematological Toxicities (grade } \geq 3 \text { ) } \\
\text { - } \quad \text { Neutropenia } \\
\text { - } \quad \text { Thrombocytopenia }\end{array}$ & \\
\hline
\end{tabular}

ANC: Absolute Neutrophil Count; Hgb: Hemoglobin; CLCr: Creatinine Clearance.

tients ( 4 male; median age 73.5 years [range 62-77]) with relapsed $\mathrm{MM}$ and ESRD requiring dialysis before receiving "lenalidomide plus dexamethasone". Median absolute neutrophil count (ANC) was $2.9 \times 10^{3} / \mathrm{uL}$ (2.8-3.3), median haemoglobin (Hgb) level was $12 \mathrm{~g} /$ $\mathrm{dL}$ (range 9.3-12.6), median platelet count was $124 \times$ $10^{3} / \mathrm{uL}$ (range 59-225). CLCr was not required. Three patients developed renal failure before MM diagnosis, 1 patient during progressive disease development and the cause of renal failure was myeloma-related. A different lenalidomide dose adjustment $(5 \mathrm{mg}$ every other day on days 1-21 of a 28-day cycle after dialysis) from the recommendations of the manufacturer was chosen due to additional fragilities (serious comorbidities, poor performance status or/and advanced age) (Table 1). The dosage of dexamethasone ( 20 or $40 \mathrm{mg}$ orally on days $1,8,15,22$ of each 28-day cycle) was modified according to patient clinical features (age, comorbidities). Two patients had antithrombotic prophylaxis with low-molecular-weight heparin as recommended in guidelines (enoxaparin $40 \mathrm{mg} / \mathrm{d}$ ) [5], 1 patient had acetylsalicylic acid $(100 \mathrm{mg} / \mathrm{d}), 1$ patient had prophylaxis with fulldose warfarin (target INR 1-3). All patients received bortezomib based-regimens as front-line therapy; only 1 patient included in our experience had previously undergone to single ASCT. Patients received a median of 1 previous therapy before starting lenalidomide (range 1-2). Until initiation of therapy with lenalidomide, renal failure persisted for a median of 28 months (range 2696). All adverse events have been graded in conformity with the National Cancer Institute Common Terminology Criteria (ver. 3.0). Responses (sCR, CR, VGPR and PR) were defined according to IMWG uniform response criteria. Response duration was calculated from the date of response to the date of relapse, disease progression or death.

\section{Results}

Only 1 patient developed grade 3 thrombocytopenia without blending events. No grade 3-4 neutropenia, severe gastrointestinal adverse events, thromboembolic complications or serious infectious episodes (bacteremias, pneumonias or sepsis) were reported. All patients achieved at least a PR; in detail, 1 patient achieved a CR, 1 a VGPR and 2 patients a PR. In accordance with previous reports, there were no significant differences in terms of overall survival and impact on response between patients who had had prior exposure to thalidomide in comparison to those who had not received thalidomide. No patients with partial or complete myeloma remission received partial or complete renal response. The median number of lenalidomide cycles was 12 (range 6-19 cycles). The median time to best response was 3.5 months (range 2-6). After 24 months, all patients are alive at the time of analysis and at time of last follow-up; 2 patients were still receiving "lenalidomide plus dexamethasone", preserving haematological response reached; 2 patients stopped treatment due to progression, with a median duration of response of 13.5 months (range 6-21). Among responding patients, median duration of response has not been reached. Lenalidomide was temporarily discontinued in only one patient due to grade 3 thrombocytopenia. The only reason to stop the treatment was disease progression.

\section{Conclusion}

Dimopoulos, et al. reported that a dose reduction of lenalidomide or interruption because of adverse events was necessary in $22 \%$ of patients with $\mathrm{MM}$ and mild or no renal impairment, $40 \%$ of patients with $\mathrm{MM}$ and moderate renal impairment and $38 \%$ of patients with $\mathrm{MM}$ and severe renal impairment; in addition, patients with severe renal impairment treated with lenalidomide have been shown to have shorter OS compared with patients with mild or no renal failure [6]. Therefore, due to substantial elimination via the kidneys, lenalidomide is recommended mainly in patients with mild-to-moderate renal impairment [7]. Particularly, in "real life", the ESRD and dialysis represent a clear exclusion criteria. In our experience, different lenalidomide dose adjustment (5 mg every other day on days 1-21 of a 28-day cycle) from 
the recommendations reported in the approved summary of product characteristics ( $5 \mathrm{mg} /$ daily) [2] is associated with a high response rate and a long duration of response. We observed a lower-than-expected incidence of haematological toxicities without an excess of prolonged cytopenia despite long-term exposure to lenalidomide. Non-haematological adverse events (nausea, diarrhea, cutaneous reactions) were usually manageable. No thromboembolic event was reported. Therefore, the treatment schedule change did not have an adverse impact on efficacy, and with appropriate dose modifications severe adverse events were largely reversible. The fact that, among responding patients, median duration of response has not been reached could shown that durability of response is comparable to those of patients with rrMM with normal renal function.

In conclusion, the combination of "lenalidomide plus dexamethasone" is an effective option for the treatment of patients with rrMM: Very severe renal failure or dialysis should not be a clear exclusion criteria. The "real life" studies helped to define the role of lenalidomide in some subpopulations of patients usually excluded from clinical trials (particularly older patients, patients living far from the hospital, patients with limited renal function): Lenalidomide remains an efficacy therapeutic strategy also for patients with light chain-induced renal failure on dialysis, but the drug dose and its mode of administration need to be adapted. An ad-hoc dose change is required for severe renal failure and patients should be closely monitored for hematologic toxicity. As indicated by this short analysis, the best-tolerated dose of lenalidomide for patients with ESRD requiring hemodialysis and additional fragilities (serious comorbidities, poor performance status or/and advanced age) should be $5 \mathrm{mg}$ every other day on days 1-21 of a 28-day cycle. Appropriate dose adjustments minimize excessive toxicities and make the combination "lenalidomide plus dexamethasone" as an effective and well tolerated therapeutic option also in very frail/unfit patients with ESRD requiring dialysis. Appropriate dose adjustments confirm significant response outcomes and significant survival benefits.
The above-mentioned results, to our knowledge, represent an additional opportunity to document the efficacy of lenalidomide reduced dosages $(5 \mathrm{mg}$ every other day on days 1-21 of a 28-day cycle Vs. $5 \mathrm{mg} /$ daily on days 1-21 of a 28-day cycle) [2] from reported recommendations for the treatment of patients with light chain-induced renal failure.

\section{Disclosure}

The authors have stated that they have no conflicts of interest. The authors are fully responsible for content of this manuscript.

\section{References}

1. Chen N, Lau H, Kong L, Zeldis JB, Knight R, et al. (2007) Pharmacokinetics of lenalidomide in subjects with various degree of renal impairment and in subjects on hemodialysis. J Clin Pharmacol 47: 1466-1475.

2. (2011) REVLIMID (Lenalidomide) [Summary of Product Characteristics]: Uxbridge, UK: Celgene Europe Limited.

3. de la Rubia J, Roig M, Ibanez A, García I, Vera JA, et al. (2010) Activity and safety of lenalidomide and dexamethasone in patients with multiple myeloma requiring dialysis: A Spanish multicenter retrospective study. Eur J Haematol 85: 363-365

4. Oehrlein K, Langer C, Sturm I, Pönisch W, Hahn-Ast C, et al. (2012) Successful treatment of patients with multiple myeloma and impaired renal function with lenalidomide: Results of 4 German centers. Clin Lymphoma Myeloma Leuk 12: 191-196.

5. Palumbo A, Rajkumar SV, Dimopoulos MA, Richardson PG, San Miguel J, et al. (2008) Prevention of thalidomide and lenalidomide-associated thrombosis in myeloma. Leukemia 22: 414-423.

6. Dimopoulos M, Alegre A, Stadtmauer EA, Goldschmidt H, Zonder JA, et al. (2010) The efficacy and safety of lenalidomide plus dexamethasone in relapsed and/or refractory multiple myeloma patients with impaired renal function. Cancer 116: 3807-3814.

7. Dimopoulos MA, Sonneveld P, Leung N, Merlini G, Ludwig $\mathrm{H}$, et al. (2016) International myeloma working group recommendations for the diagnosis and management of myeloma- related renal impairment. J Clin Oncol 34: 15441557. 